\title{
Dirofilariasis involving the oral cavity: report of the first case from South America
}

\author{
Laudenice Lucena Pereira ${ }^{[1]}$, Ricardo Della Coletta ${ }^{[2]}$, Larissa Cavalcanti Monteiro $^{[1]}$, \\ Victor Yuri Nicolau Ferreira ${ }^{[1]}$, Jorge Esquiche Leon $^{[3]}$ \\ and Paulo Rogério Ferreti Bonan ${ }^{[1]}$
}

[1]. Faculdade de Odontologia, Universidade Federal da Paraíba, João Pessoa, Paraíba, Brasil. [2]. Faculdade de Odontologia, Universidade Estadual de Campinas, Piracicaba, São Paulo, Brasil. [3]. Faculdade de Odontologia, Universidade de São Paulo, Ribeirão Preto, São Paulo, Brasil.

\begin{abstract}
Oral dirofilariasis is very rare with non-specific clinical manifestations. Here, we report the case of a 65 -year-old South American woman with a submucosal nodule on her right buccal mucosa. The nodule was slightly tender and painful. Differential diagnoses included mesenchymal (lipoma or fibrolipoma, solitary fibrous tumor, and neurofibroma) or glandular benign tumors (pleomorphic adenoma) with secondary infections. We performed excisional biopsy. A histopathological examination revealed a dense fibrous capsule and a single female filarial worm showing double uterus appearance, neural plaque, well-developed musculature and intestinal apparatus. Dirofilariasis was diagnosed, and the patient was followed-up for 12 months without recurrence.
\end{abstract}

Keywords: Pathology. Dirofilariasis. Dentistry. Diagnosis.

\section{INTRODUCTION}

Filariasis is a zoonosis that occurs worldwide but is more common in countries with tropical and subtropical climates ${ }^{(1)}$. It is caused by nematodes belonging to the genus Dirofilaria, and is transmitted to humans via bites of Aedes, Culex, and Anopheles mosquitoes ${ }^{(2)}$. Dirofilaria repens, Dirofilaria immitis, Dirofilaria ursi, and Dirofilaria tenuis are the species known to affect dogs, foxes, cats, raccoons, and bears, which serve as the intermediate hosts ${ }^{(2)}$.

Dirofilaria repens, one of the more common species, can cause submucosal nodules on the arms, legs, head, and even the eyes of humans; these nodules develop as an inflammatory reaction to the presence of the parasite in the tissue ${ }^{(3)}$. Intraoral involvement is very rare, and diagnosis is confirmed based on identification of the parasite in the nodule under microscopy ${ }^{(4)}$. Definitive treatment includes surgical removal, and in some cases, medications such as diethylcarbamazine, ivermectin, and albendazole ${ }^{(1)}$. In this study, we describe a rare case of dirofilariasis affecting the oral mucosa. To the best of our knowledge, this case is the first to be reported from Brazil and from South America.

Correspondence author: Prof. Paulo Rogério Ferreti Bonan. Faculdade de Odontologia/UFPB. Cidade Universitária, Castelo Branco, 58051-800 João Pessoa, Paraíba, Brasil.

Phone: 5583 8882-7842

e-mail: pbonan@yahoo.com; nicelucena@hotmail.com

Received 23 January 2015

Accepted 23 March 2015

\section{CASE REPORT}

A feoderma 65-year-old woman was referred to our hospital by the public health unit. She complained of swelling and pain on the right side of her buccal mucosa. She was unaware of when the nodule first appeared, and hence could not tell when the swelling exacerbated. Her dentist had prescribed antibiotics, anti-inflammatories, and analgesics. Despite these medications, her symptoms persisted and worsened. Our patient lived in a very crowded community with poor sanitary conditions and constant warm and humid climate. She had good general health with no gastrointestinal complaints and she did not consume tobacco. General physical examination revealed no relevant findings or lymphadenopathy.

Extraoral clinical examination revealed normal structures without tumefactions. Intraoral examination revealed a movable tender mass covered by normal mucosa on the right side of the buccal mucosa (Figure 1, left). Differential diagnosis included mesenchymal benign tumors (such as lipoma or fibrolipoma, solitary fibrous tumor, and neurofibroma) or glandular benign tumors (such as pleomorphic adenoma) with inflammatory reactions, including secondary infections. Excisional biopsy was performed. Periodic acid-Schiff staining was carried out to evaluate secondary fungal infections; no evidence of yeasts or hyphae was found. Histological examination revealed a dense fibrous capsule containing an infiltrate of mixed inflammatory cells-lymphocytes, macrophages, eosinophils, and neutrophils. Within the infiltrate, we found a single female worm with numerous larval forms. Higher magnification revealed a thick cuticle, double uterus, neural plaque, and centrally placed 


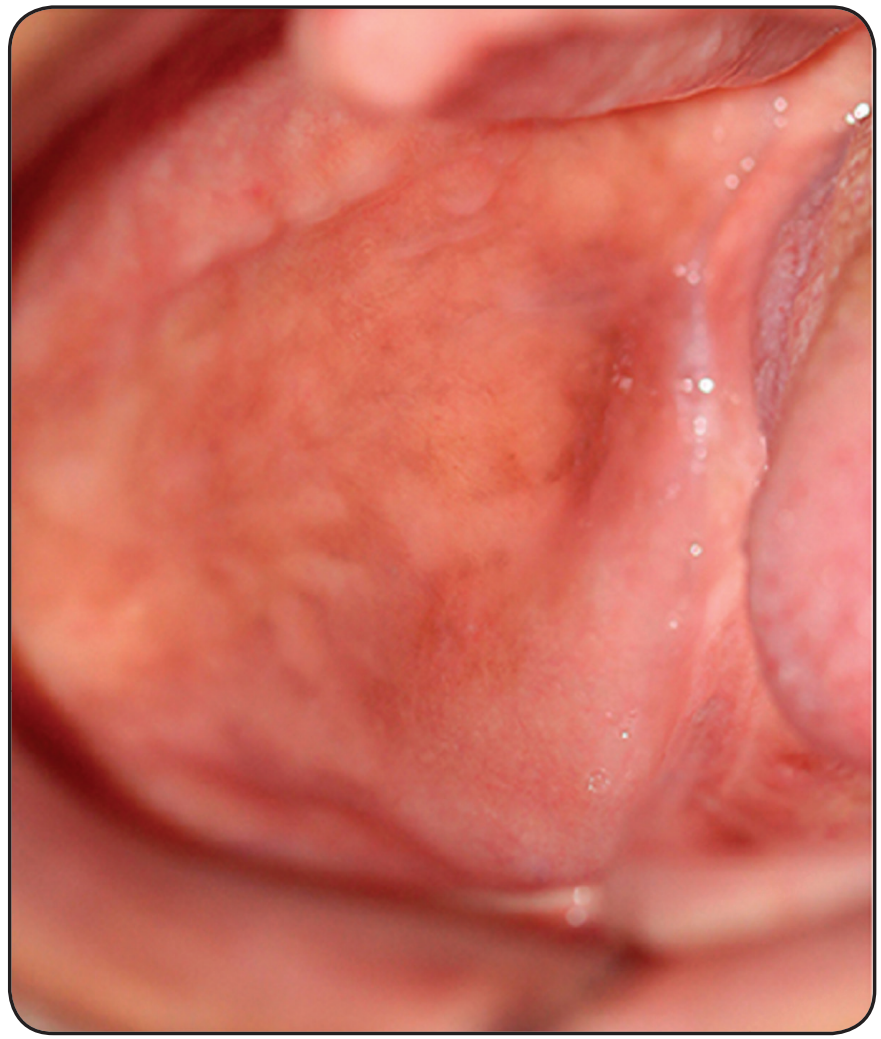

FIGURE 1 - Symptomatic well-defined nodule on the right side of the buccal mucosa.

intestinal tubule within the well-developed musculature, characteristic of the Dirofilaria species (Figure 2). With these microscopy data and considering the poor sanitary conditions, we performed blood tests and the results were unremarkable with respect to systemic infections. Parasitological examination, too, did not reveal eggs or larvae of parasites. Thus, a definitive diagnosis of dirofilariasis was made. None additional treatment was required due to be a local manifestation. The patient was followed-up and 12 months after diagnosis she presented with good general health and absence of recurrence (Figure 3).

\section{DISCUSSION}

Although heartworm disease occurs worldwide, it is rare in humans. Dirofilaria species are natural parasites of mammals and can be accidentally transmitted to humans via bites of zooanthrophilic mosquitoes carrying the infectious larvae. Infectious larvae acquired during a blood meal from host animals infected with the Dirofilaria sp. Humans are not ideal hosts, as in the human body, Dirofilaria species develop into worms that do not reproduce or release microfilariae. Although nearly 40 Dirofilaria species have been identified, only a few have been reported to cause human infections ${ }^{(1)(2)}$. In our case, the genus could be identified only because we performed a morphological evaluation using microscopy, which is not the gold standard for species classification ${ }^{(1)}$.

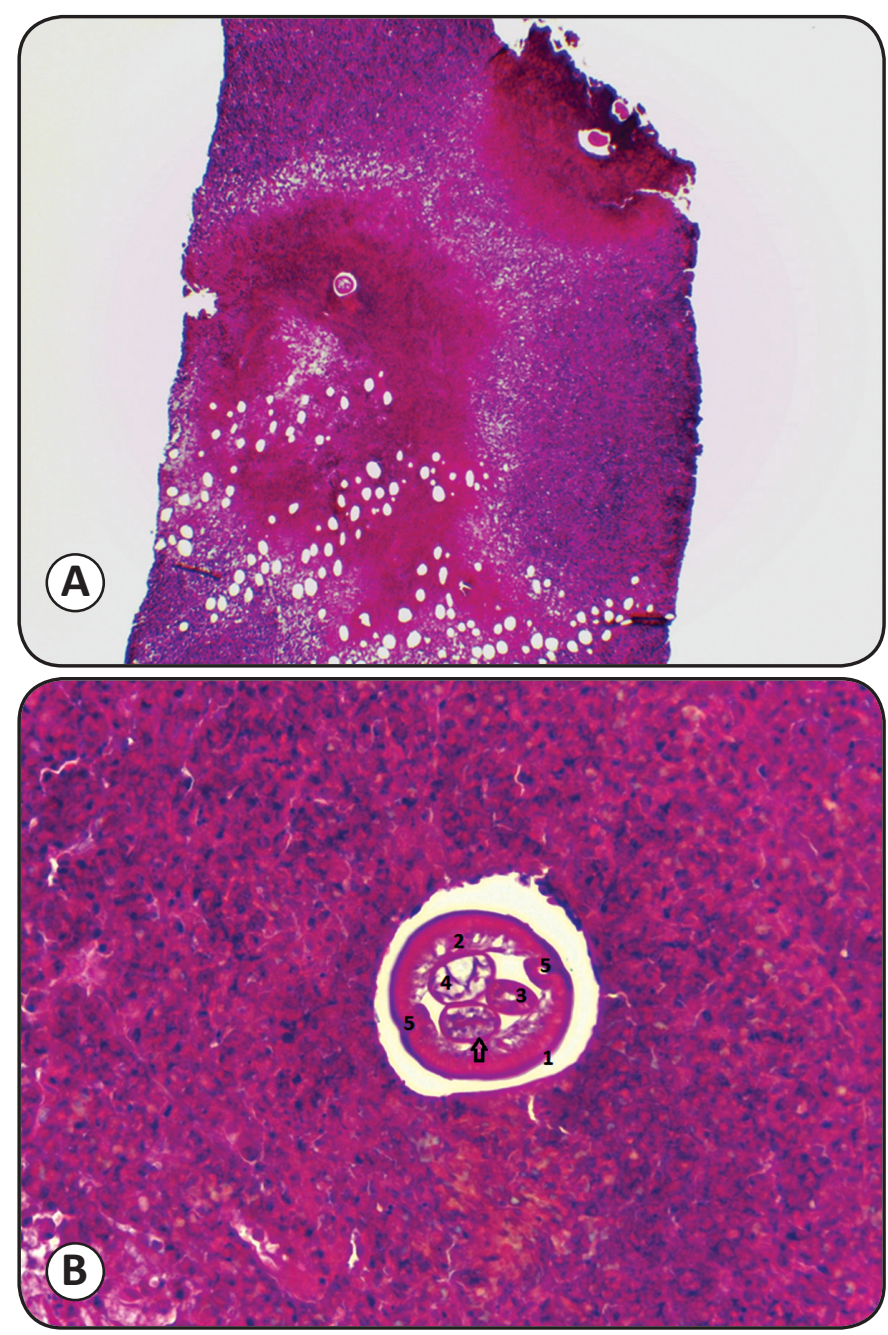

FIGURE 2 - (A) Mixed inflammatory infiltrate and female nematoid body placed inside the tissue (HE, 100X). (B) Thick cuticle 1: developed musculature 2: double uterus 3: with eggs (arrow), intestinal apparatus 4: and neural plaque 5: revealing Dirofilaria species (HE, 400X).

It is assumed that the increase in cases of heartworm disease in humans is caused by climate change with higher temperatures favoring the survival of microfilariae and by global tourism increasing that can causes infection by infected carnivores in certain regions of the world ${ }^{(3)}$. Knowledge of endemic areas with poor vector control owing to improper sanitation and overpopulation is often helpful in diagnosis ${ }^{(1)}$. In the current case, the patient did not report going abroad but she lived in a very populated region with high temperatures and poor sanitary conditions.

The clinical manifestations of dirofilariasis include nodules in the subcutaneous tissues, muscles, and visceral organs. The duration between growth of the paras causing infection to the detection of the nodule is approximately 6 months. The parasite is restricted within the inflammatory subcutaneous tissue node or other tissues, and can survive for months or even years. Some cases of dirofilariasis are asymptomatic; nevertheless, cases with itching, arthritis, edema, and subcutaneous swelling have been 


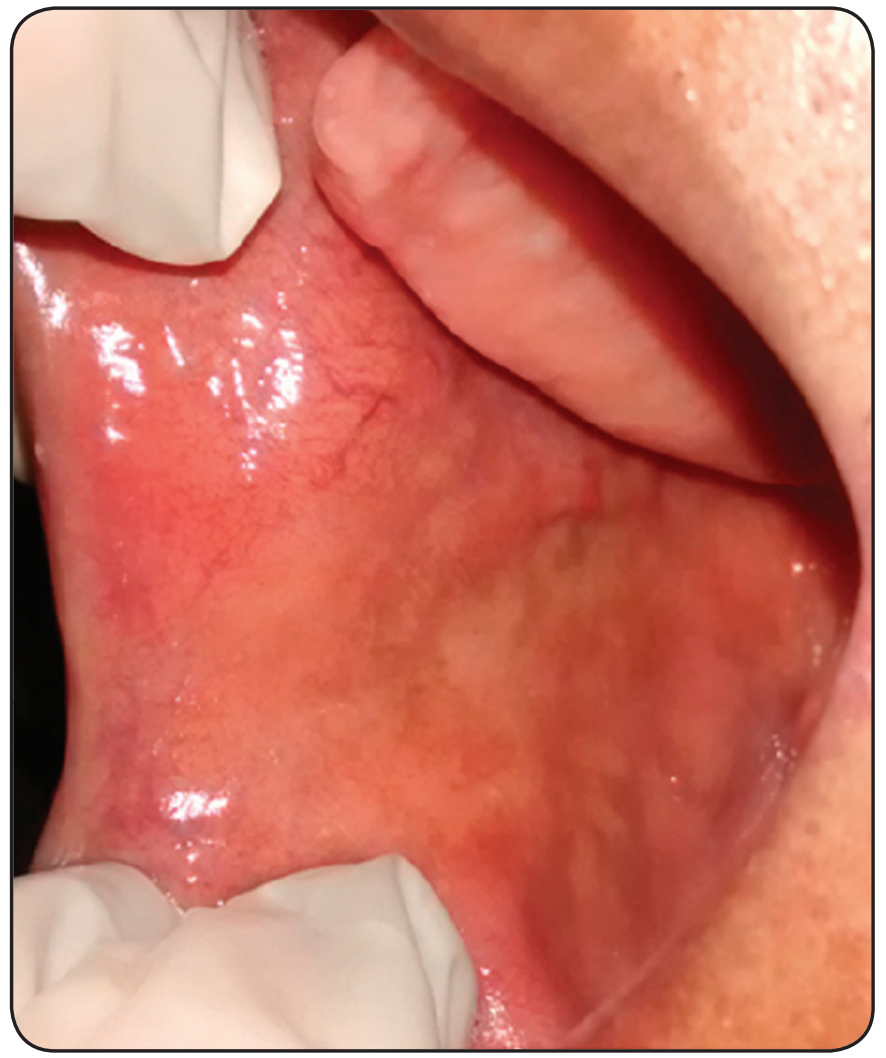

FIGURE 3 - Buccal mucosa showing no recurrence after 12 months of follow-up.

described ${ }^{(4)}$. In this case, the patient was symptomatic and an intense inflammatory reaction was observed. Our differential diagnoses included mesenchymal benign tumors (such as lipoma or fibrolipoma, solitary fibrous tumor, and neurofibroma) or glandular benign tumors (such as pleomorphic adenoma) with inflammatory reactions, including secondary infections. Some of these lesions were also included as differential diagnoses in a previous case of dirofilariasis affecting the oral mucosa ${ }^{(1)}$.

Few studies have reported the occurrence of dirofilariasis involving the head and neck. A study by Latifoglu et al. ${ }^{(5)}$ conducted in 2002 cited the presence of a nematode clearly identified by histopathology after excisional biopsy of a mass on the right cheek, lateral to the masseter muscle. Additional radiographic and ultrasonographic examinations of the site were performed to rule out any connection with the parotid gland. A more recent study conducted in 2014 by Friedrich et al. ${ }^{\left({ }^{6}\right.}$ verified the presence of Dirofilaria infection in a 40-year-old patient without apparent symptoms. The only clinical feature was the presence of a swelling in the left temporal muscle. This patient had travelled to Sri Lanka 8 months prior to the onset of the swelling. In addition, he had travelled to the Far East and the Indian subcontinent few years ago. This information is relevant since the occurrence of dirofilariasis in humans is more common in those regions of the world. In 2013, Kurup et al. ${ }^{(1)}$ described the case of a 54-year-old female school teacher who complained of intermittent swelling inside her mouth on the left side. Dirofilariasis was diagnosed after microscopic examination, and the buccal mucosa showed features similar to those observed in the current case.

A detailed monitoring of the patient is necessary for diagnosis, and additional tests are needed to confirm the diagnosis ${ }^{(5)}$. Blood and stool tests for parasites could be performed. Excisional biopsy for histologic evaluation of the nodule was essential for accurate diagnosis. Aside from being a diagnostic procedure, excisional biopsy is a treatment method. The histopathology diagnosis could reveal the presence of a double uterus, possibly containing many larval forms, neural plaque, gut apparatus, and a thick cuticle, confirming the diagnosis of dirofilariasis ${ }^{(1)}$. The thick cuticle and developed musculature are characteristic of Dirofilaria species $^{(7)}$. The treatment could involve surgical removal, and in some cases, medications such as diethylcarbamazine, ivermectin, and albendazole ${ }^{(1)}$. In our case, we only observed the focal infection without systemic involvement. After removal of the nodule, the patient was followed-up for 12 months. She was in good health and had no recurrence.

Dirofilariasis involving the oral cavity is very rare. However, it can be found in countries with high temperatures and in populated communities with poor sanitary conditions. Health professionals need to be aware of the need for detailed examination and must consider infections caused by parasites in their differential diagnoses. If required, ancillary tests must be performed to aid diagnosis.

\section{REFERENCES}

1. Kurup S, Veeraraghavan R, Jose R, Puthalath U. Filariasis of the buccal mucosa: a diagnostic dilemma. Contemp Clin Dent 2013; 4:254-257.

2. Orihel TC, Eberhard ML. Zoonotic filariasis. Clin Microbiol Rev 1998; 11:366-381.

3. Otranto D, Dantas-Torres F, Brianti E, Traversa D, Petrić D, Genchi C, et al. Vector-borne helminths of dogs and humans in Europe. Parasit Vectors 2013; 6:16.

4. Campos JRM, Barbas CSV, Filomeno LTB, Fernandez A, Minamoto H, B Filho JV, et al. Human pulmonary dirofilariasis. Analysis of 24 cases from São Paulo, Brazil. Chest 1997; 112: 729-733.

5. Latifoglu O, Ozmen S, Sezer C, Yavuzer R, Altintas K, Uluoglu O. Dirofilaria repens presenting as a premasseteric nodule. Oral Surg Oral Med Oral Pathol Oral Radiol Endod 2002; 94:217-220.

6. Friedrich RE, Heiland M, Burchard G, Racz P, Zustin J, Hagel C. Human Dirofilaria repens infection of the zygomatico-temporal region. J Craniomaxillofac Surg 2014; 42:612e615.

7. Popescu I, Tudose I, Racz P, Muntau B, Giurcaneanu C, Poppert S. Human Dirofilaria repens infection in Romania: a case report. Case Rep Infect Dis 2012; 2012:472-976. 